Aims. This study looked at the differences in the length of hospital stays in number of days, 12 months before and after starting on Lurasidone, in patients with psychosis.

Method. A retrospective review of medical records between 2016 and 2019 of patients with psychosis due to all causes at a First Episode Psychosis service in the United Kingdom was performed. Most common side effects, duration of Lurasidone treatment and reasons for stopping Lurasidone were recorded. The length of hospital stays (in number of days) before and after being started on Lurasidone of those had taken Lurasidone for at least 12 months were compared using a paired t-test.

Result. $43(\mathrm{n}=43)$ patients had taken Lurasidone at some point during the study period with a mean age of 30.48 years and a male: female ratio of 1.4:1. The average duration of treatment was 327 days. The most common reported side effects were sedation (16\%), nausea (7\%) and tardive dyskinesia (7\%). Among these 43 patients, 19 patients (44\%) tolerated and were on Lurasidone for at least 12 months with a mean age of 30.42 and a male: female ratio of $0.42: 1$. Of these 19 patients, the total number of days of hospital stays within 12 months before and after Lurasidone initiation was 1179 days $($ mean $=62.05)$ and 242 days $($ mean $=16.47)$ respectively. The paired t-test showed a significant reduction in the average length of hospital stays in these patients within 12 months after Lurasidone initiation ( $\mathrm{p}=$ 0.0466).

Conclusion. Patients with psychosis who were on Lurasidone had a statically significantly reduction in the length of hospital stays within 12 months of medication initiation; up to $44 \%$ tolerance rate, with better tolerance in female patients and the most common side effects being sedation, nausea, tardive dyskinesia.

\section{Evidence base for psychological treatment of personality disorder - a narrative review}

Caoimhe Ni Shuilleabhain

Camden and Islington NHS Trust

doi: 10.1192/bjo.2021.739

Aims. This review critically appraises the up-to-date evidence base for psychological treatment of PD.

Background. The prevalence rate of any personality disorder (PD) in the general population has been estimated to be as high as $12 \%$ rising to over $70 \%$ in prison settings. PD is known to carry significant psychosocial and health burdens with increased mortality, increased suicide, increased substance misuse, increased crime, reduced capacity to work, poorer outcomes for comorbid mental disorders, dysfunctional engagement with services, and high economic costs through a high utilisation of healthcare systems. In the $1990 \mathrm{~s}$ several manualised treatment strategies emerged, specifically for borderline PD. These include dialectical behaviour therapy, cognitive therapy, cognitive analytic therapy, mentalization-based therapy, transference-focused psychotherapy, and schema-focussed therapy.

Method. Using relevant search criteria, literature was identified through a search of the following databases: PubMed, EMBASE, and PsycINFO. Data were appraised and synthesised to provide a comprehensive overview of the current evidence base for psychological treatment of PD.

Result. The DSM-V defined Cluster B borderline PD has received the majority of attention. Increasing attention has been paid in recent years to the Cluster B antisocial PD. Cluster A (Paranoid, Schizoid, Schizotypal) and Cluster C PDs (Avoidant, Dependent, Obsessive Compulsive) have received relatively little attention with few studies to draw upon regarding the effectiveness of therapy.
The remaining Cluster B personality disorders (Narcissistic and Histrionic) have been criticised as having poor construct validity, with a lack of rigorously designed treatment trials.

A number of treatment protocols have gained empirical support. However, of those that have empirical support, there appears to be little demonstrable evidence to suggest superiority of any one of the evidence-based interventions over another. While specialised therapies are more efficacious than "treatment as usual" or treatment delivered by expert clinicians, when specialised therapies are compared with well-specified manualised general psychiatric care tailored to personality disorder, the results are different, with little consistent evidence demonstrating the superiority of specialised therapies.

Conclusion. Current evidence suggests that individual therapies do not differ substantially from each other or from structured clinical care that relies on generic change factors. This is in keeping with established psychotherapy outcome literature. Current evidence would indicate that common features across the proven treatment strategies should be emphasised and implemented well. There may be justification for added interventions from specific treatment modalities targeted to specific patient problems.

\section{Autism in girls and the pre-referral environment}

Peter Nussbaum

NHS Lothian

doi: 10.1192/bjo.2021.740

Aims. This literature review sought to identify and highlight any sex specific factors in the diagnosis of autism spectrum conditions during the pre-referral period which might affect diagnosis rates in ASD in girls. The null hypothesis was that there are no sex specific factors that affect referral and diagnosis of ASD in girls.

Background. Historically, boys are diagnosed with ASD more than girls but rates vary depending on clinical population characteristics. Diagnosis trends continue to demonstrate a large male excess. The concept of autism as a predominantly male condition has been challenged and there is increased focus on females with high functioning autism who are not being detected as easily.

Various theories exist as to why this is the case.

There are high rates of suicidality in ASD and risk of death by suicide is higher in ASD women (the reciprocal of the suicide rates in general population where more men complete suicide). Women with high functioning autism represent an at risk group. Undetected autism in females may be complicated by 'camouflaging' or masking of symptoms which puts a large strain on individuals functioning and mental health. Costs to society and the individual are large.

However, early identification and intervention improves outcomes such as activities of daily living and social behaviours.

Method. An electronic literature search was completed using MEDLINE, PsycINFO and EMBASE in November 2018. Key

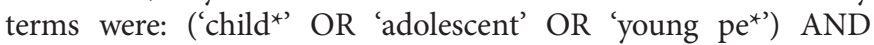
('ASD' OR 'autism' OR 'asperger*' OR 'high functioning ${ }^{\star}$ ' OR 'PDD' OR 'Pervasive developmental ${ }^{\star}$ ) AND ('girl' ${ }^{\star}$ OR 'sex' OR 'gender'). Papers were excluded on a number of grounds.

Result. 11 papers were included in the review from an initial 2823 abstracts.

Conclusion. A number of papers highlighted important learning points. Some of the more original conclusions included that we require more studies comparing populations of girls with ASD to high risk, high functioning girls and female controls to clarify features particular to the 'female phenotype'. Delays in diagnosis in girls appears to pre-date assessment so further thought on how to 
educate and support referral sources (caregivers and schools) on how to identify girls with autism is recommended and would be informed by further research focus on the previous point. Active case ascertainment should be considered in future research and follow-up of girls who do not receive a diagnosis at initial assessment were additional learning points that came from the review.

\section{Quality of sleep and burnout among undergraduate medical students at the university of Nairobi, Kenya \\ Linda Nyamute ${ }^{1 \star}$, Muthoni Mathai ${ }^{2}$ and Anne Mbwayo ${ }^{3}$ \\ ${ }^{1}$ University of Nairobi; ${ }^{2}$ Consultant Psychiatrist and Senior lecturer, University of Nairobi and ${ }^{3}$ Clinical psychologist and lecturer, \\ University of Nairobi \\ ${ }^{*}$ Corresponding author.}

doi: 10.1192/bjo.2021.742

Aims. The main objective was to determine whether quality of sleep is associated with burnout among undergraduate medical students at the University of Nairobi.

The null hypothesis in our study population was; 'There is no significant association between poor sleep quality and burnout'. Background. In a pressure prevailing environment, medical students find themselves in a vicious cycle of cutting down on sleep in attempts to cope and adjust to increasing workloads. Students with poor sleep quality have been found to perform worse in their board exam and have strained social engagements. Ultimately, this chronic sleep deprivation may lead to burnout which may cause diminished sense of accomplishment and impaired professional conduct, that may be carried on to the career as a physician. High levels of burnout have been associated with suicides.

Method. The sample size obtained was 384 and participants were selected by a mixed sampling method. Data collection was through self-administered questionnaires. Scales used for this study were the Pittsburg Sleep Quality Index(PSQI) and the Oldenburg Burnout Inventory(OLBI).

Ethical considerations were adhered to and approval obtained from the Kenyatta National Hospital-University of Nairobi (KNH-UON) Ethics Board. Data entry and analysis was by SPSS v23. Data from 336 questionnaires were deemed fit for analysis.

Result. With a response rate of $87.5 \%$, the prevalence of poor sleep quality and burnout were $69.9 \%$ and $74.7 \%$ respectively. There was a significant positive association between poor sleep quality and female gender, clinical years of study, living with family, poorly perceived socio-economic state and poor subjective academic performance. In addition, being female, younger, preclinical years, living independently off-campus and poor subjective academic performance were significantly associated with higher levels of burnout.

Burnout had a significant correlation with poor sleep quality. Daytime functioning, a component of sleep quality had the highest correlation with components of burnout, disengagement and exhaustion. Overall, $57 \%$ of the respondents had both poor sleep quality \&burnout, while only $12 \%$ were good sleepers with no burnout. Furthermore, having poor sleep increased the risk of having burnout by 2.8 times. It is crucial that students adopt better sleeping habits to reduce the risk of burnout.

Conclusion. With the high prevalence of poor sleep quality and burnout, peer-support groups and peer-led mentorship programs are recommended within this population to help deal with expectations, challenges and difficulties encountered within the course of medical education, in addition to preparing for the early future careers.
What is the prevalence of self-harming and suicidal behaviour in under 18s with autism spectrum disorder, with or without an intellectual disability?

Rosalind Oliphant*, Eleanor Smith and Victoria Grahame

Cumbria, Northumberland, Tyne And Wear NHS Foundation Trust ${ }^{\star}$ Corresponding author.

doi: 10.1192/bjo.2021.743

Aims. The aims of this systematic review are to summarise data on the prevalence of suicidal behaviours and self-harm in under $18 \mathrm{~s}$ with Autism Spectrum Disorder (ASD) and consider the impact of Intellectual Disability (ID). It was hypothesised that the prevalence of these behaviours may be higher in under $18 \mathrm{~s}$ with ASD than in the general population.

Background. In the general population, rates of self-harm and suicide in under $18 \mathrm{~s}$ are of increasing concern. Whilst there is an emerging evidence base considering suicidality in autistic adults, less in known about the experience of under 18s. There has been very little research focused on how self-harm seen within the general population presents in the context of ASD and whether it continues to be a predictor of future suicidal behaviour. This may be partly due to self-harm being considered alongside Self-Injurious Behaviours (SIB), which have long been recognised as part of the clinical presentation of ASD and may have other functions (e.g. fulfilling sensory stimulation needs).

Method. A systematic literature search was conducted in line with PRISMA guidelines. For this review, all papers that included data on prevalence of self-harm and/or suicidal behaviours in under $18 \mathrm{~s}$ with ASD were included. Studies that only reported on the prevalence of the broader entity of SIB (characterised as stereotypic or habitual) were excluded.

Result. 338 papers were initially identified and 9 met eligibility criteria. There was considerable variation in how different aspects of self-harm and suicidal behaviours were addressed between groups and also between population samples, making it difficult to generalise the findings. The prevalence of self-harming and suicidal behaviours ranged from $7 \%$ to $73 \%$, indicating that this is a clinically significant problem for this patient group. The only study that considered the impact of co-existing ID did not identify significant differences between groups (ID vs no ID).

Conclusion. There was variation in the reported prevalence rates but results suggested that rates of both self-harm and suicidal behaviour may be elevated in under $18 \mathrm{~s}$ with ASD compared to the general population. This is in keeping with literature relating to autistic adults but in contrast to conclusions of a previous systematic review. This review highlights the need for further research to explore the experience of self-harm and suicidal behaviour in autistic children and young people.

\section{A survey into the influence of dopaminergic drug exposure on 'sense of presence' symptoms in patients with parkinson's disease

\author{
Emma Padfield*, Hannah Potticary and Tim Segal \\ Kings College London, South London and Maudsley NHS \\ Foundation Trust \\ ${ }^{\star}$ Corresponding author.
}

doi: 10.1192/bjo.2021.744

Aims. The first objective was to estimate prevalence of sense of presence (SoP) experiences in patients with Parkinson's Disease (PD), including whether onset was prior to or after commencing 